\title{
Band Edge Energies Pinning in Magnetic Field in Semimagnetic $\mathrm{Cd}_{0.7} \mathrm{Mn}_{0.3} \mathrm{Te}$
}

\author{
L. Barauskaité丶 $\dot{E}^{a *}, \mathrm{R} . \mathrm{Brazis}^{a}, \mathrm{~V} . \mathrm{IVAnOV}^{b}$ And M. Godlewski $^{b}$ \\ ${ }^{a}$ Center of Physical Sciences and Technology, A. Goštauto 11, 01108 Vilnius, Lithuania \\ ${ }^{b}$ Institute of Physics, Polish Academy of Sciences, al. Lotników 32/46, 02-668 Warsaw, Poland
}

(Received November 12, 2010; in final form July 8, 2011)

\begin{abstract}
Results of $\mathrm{Cd}_{0.7} \mathrm{Mn}_{0.3}$ Te magneto-photoluminescence investigations are analyzed. Photoluminescence was measured in a Voigt geometry at the temperature of $1.6 \mathrm{~K}$. Two models describing radiative transition energy are compared with the experimental results of $\mathrm{Cd}_{0.7} \mathrm{Mn}_{0.3}$ Te luminescence. The energy of recombination transition, employing the complete form of band states splitting description (term due to the Landau splitting included), shows deviation from experiment at $B>2 \mathrm{~T}$. Two possible reasons of discrepancies from the experiment exchange interaction constants dependence on magnetic field or band edge energies pinning in magnetic field are discussed. Band edge energies pinning in magnetic field is related to the hybridization of Mn $3 d^{5}$ levels with the band states of the host. We determine the corrected values of exchange interaction constants $\left(N_{0} \alpha-N_{0} \beta / 3\right)$ at magnetic fields $B<5 \mathrm{~T}$.
\end{abstract}

PACS: 78.55.Et, 75.50.Pp, 71.70.-d

\section{Introduction}

From the early stages of investigation of semimagnetic semiconductors (SMSCs) the interpretation of magneto-optical experiments of semiconducting compounds with magnetic ions was based on the $s, p-d$ exchange interaction theory [1-3]. Authors of these references succeeded to describe the enhanced Zeeman splitting of band edge states by independently measured values of magnetization $\left\langle S_{z}\right\rangle$ and exchange interaction constants $N_{0} \alpha$ and $N_{0} \beta$. These constants describe the $s-d$ and $p-d$ exchange, respectively. The $N_{0} \alpha$ and $N_{0} \beta$ values were independent of concentration of magnetic impurity $x$ and magnetic field. In the original approach introduced in [1-3] the influence of magnetic field on the band states, i.e., the Landau quantization, was neglected. This was justified for large-gap semimagnetic semiconductors with parabolic bands and large effective masses [1-4]. This simplified model (without cyclotron term $\hbar \omega_{\text {ce }}$ ) succeeded well to describe the magneto-optical results.

In the present magneto-photoluminescence (magneto-PL) study of $\mathrm{Cd}_{0.7} \mathrm{Mn}_{0.3} \mathrm{Te}$ we first checked the influence of the Landau splitting term $\hbar \omega_{\text {ce }}$. Surprisingly, we obtained that in this case to achieve the coincidence with the experiment, the exchange interaction constants $N_{0} \alpha$ and $N_{0} \beta$ should depend on magnetic field. As the simplified model of band edge states splitting (approximation with the $s, p-d$ exchange interaction term only) holds well, the obtained contradiction is analyzed in our work.

Since dependence of exchange interaction constants $N_{0} \alpha$ and $N_{0} \beta$ on magnetic field is rather unexpected, we

* corresponding author; e-mail: lb@pfi.lt analyse the alternative explanation of the obtained result, i.e., that the band edge energies values of $\mathrm{Cd}_{0.7} \mathrm{Mn}_{0.3} \mathrm{Te}$ are pinned in magnetic field. Possible explanation, based on the influence of $\mathrm{Mn}$ ions on the band structure of host semiconductor, is proposed in this work.

\section{Experimental details}

$\mathrm{Cd}_{0.7} \mathrm{Mn}_{0.3}$ Te crystals were grown by the modified Bridgman method. Photoluminescence (PL) was studied in a magnetic field up to $7 \mathrm{~T}$ at the temperature of $1.6 \mathrm{~K}$. For PL excitation we used the $\operatorname{Ar}$ laser $(\lambda=488 \mathrm{~nm})$ line, directed at the angle of $45^{\circ}$ on the cleaved surface of the crystal. Magnetic field was perpendicular to the direction of the incident beam (the Voigt geometry). Further details of the experimental setup are given in [5]. The $\sigma$ and $\pi$ components of $\mathrm{Cd}_{0.7} \mathrm{Mn}_{0.3} \mathrm{Te}$ PL taken in the backward (bcwd) direction (relative to the incident beam) are presented in Fig. 1.

\section{Discussion}

\subsection{Do exchange interaction constants $\left(N_{0} \alpha-N_{0} \beta / 3\right)$ depend on magnetic field?}

To interpret the PL results of $\mathrm{Cd}_{0.7} \mathrm{Mn}_{0.3}$ Te luminescence in a magnetic field, we first analyze the details of the band states splitting in a magnetic field. We assume that in semimagnetic semiconductors selection rules for allowed transitions between the band edge states split by magnetic field are the same as for nonmagnetic semiconductors (Fig. 1). Experimentally determined energies of luminescent transitions indicate that in the light emission process only transitions from the lowest level of a conduction band are involved. With the calculated $\left\langle S_{z}\right\rangle$ 
value we can evaluate the energies of related transitions in a magnetic field. The aim of such calculations is to explain the experimentally detected narrowing of the effective band gap, i.e., the red shift of PL maximum with the increase of a magnetic field (Fig. 2) [5]. To describe the observed red shift of the main luminescence maximum in magnetic field we discuss possible descriptions of band splitting in SMSCs in magnetic field.

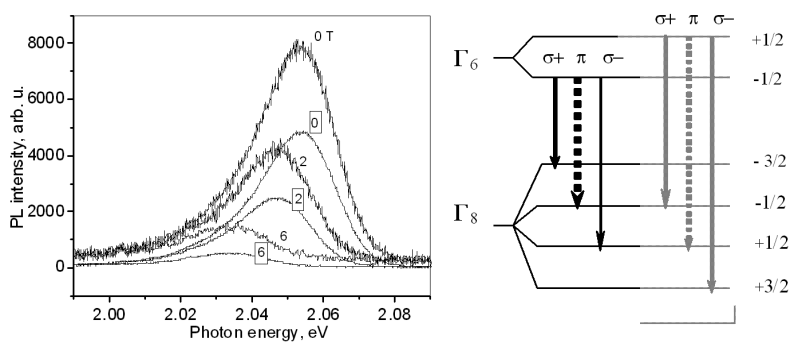

Fig. 1. $\pi$ and $\sigma$ components of $\mathrm{Cd}_{0.7} \mathrm{Mn}_{0.3} \mathrm{Te}$ crystal photoluminescence measured in bcwd geometry ( $\square$ marks the $\sigma$ spectra, the numbers show magnetic field values in $\mathrm{T}$ ). Zeeman splitting scheme and selection rules of radiative transitions in a magnetic field are also shown.

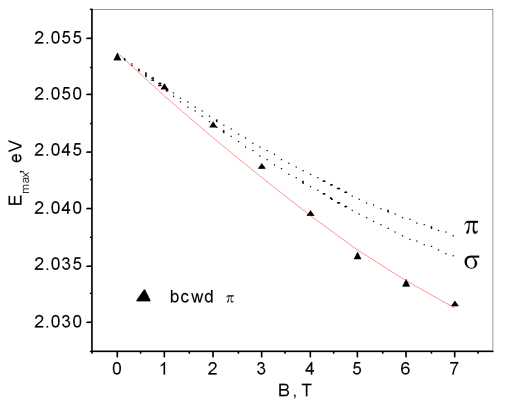

Fig. 2. $\mathrm{Cd}_{0.7} \mathrm{Mn}_{0.3}$ Te luminescence maximum spectral position in a magnetic field ( $\pi$ component, bcwd); $\Delta$ experiment, solid line - recombination energy evaluated, assuming band states splitting approximation with the $s, p-d$ exchange interaction term; dashed lines $-\pi$ and $\sigma$ energies of recombination transitions (cyclotronic term included in band states splitting description).

Experimentally detected energy of recombination emission was analyzed according to the scheme of Fig. 1 and employing two usually used approaches to describe splitting of band states in a magnetic field:

1. The complete description of band edge states splitting (when the Landau splitting term is included). In this case the energy of an electron in a magnetic field in the conduction band is described

$$
\begin{gathered}
E_{n}(\uparrow \downarrow)=E_{\mathrm{g}}+\left(n+\frac{1}{2}\right) \hbar \omega_{\mathrm{ce}} \\
\pm \frac{1}{2}\left(g \mu_{\mathrm{B}} B+x N_{0} \alpha\left\langle S_{z}\right\rangle\right),
\end{gathered}
$$

where $\omega_{\text {ce }}$ is cyclotron frequency, $x$ is the Mn mole fraction, $N_{0} \alpha$ is the $s-d$ exchange interaction constant, $\left\langle S_{z}\right\rangle$ is the thermodynamical average of $\mathrm{Mn}^{2+}$ ion spin, $g$ is the Landé factor of electron, $\mu_{\mathrm{B}}$ is the Bohr magneton.

2. Band edge states splittings approximation with the $s, p-d$ exchange interaction terms only, described by $[6,7]$ :

$$
\begin{aligned}
& \Delta E_{\mathrm{c}}=-x N_{0} \alpha\left\langle S_{z}\right\rangle m_{j}, \quad m_{j}= \pm 1 / 2 \quad \text { and } \\
& \Delta E_{\mathrm{v}}=-(1 / 3) x N_{0} \beta\left\langle S_{z}\right\rangle m_{j}, \quad m_{j}= \pm 1 / 2 ; \pm 3 / 2,
\end{aligned}
$$

where $N_{0} \beta$ is the $p-d$ exchange interaction constant, $m_{j}$ is magnetic quantum number.

For large-gap semimagnetic semiconductors with parabolic bands and large effective masses, when the Landau and intrinsic spin splittings are considerably smaller than the $s, p-d$ exchange interaction term, the band states splitting approximation with $s, p-d$ exchange interaction term only is used [1-4]. We notice again that this approach was successfully applied to analyze magneto-optical results of SMSCs.

Many authors $[1,2,6]$ agree that the shortened form of band states splitting description is sufficient for the analysis of experimental results, but the accuracy of the applied approximation in various magnetic field ranges has been rarely discussed. Relying on performed analysis of $\mathrm{Cd}_{0.7} \mathrm{Mn}_{0.3} \mathrm{Te}$ luminescence results, the accuracy of both possible band states splitting descriptions is evaluated by us in weak and stronger magnetic fields. The PL spectral shift towards lower energies in magnetic field for $\pi$ polarized light is in good but not precise agreement with the approximate model discussed above, when the Landau splittings are not included in the calculations (Fig. 2).

In the case of $\mathrm{Cd}_{0.7} \mathrm{Mn}_{0.3} \mathrm{Te}$ Landau splitting term $\hbar \omega_{\text {ce }}$ amounts for the $20 \%$ of spin-splitting due to the $s, p-d$ exchange. For that reason in analysis of $\mathrm{Cd}_{0.7} \mathrm{Mn}_{0.3} \mathrm{Te} \mathrm{PL}$ we took into account the cyclotronic term and obtained the unexpected deviation from the experiment (Fig. 2). The improved agreement with the experimental data was obtained only in the low field region $B<2 \mathrm{~T}$. Experimental results indicate that band splitting approximation by the exchange interaction term correctly describes the effective band gap narrowing only at fields $B>4 \mathrm{~T}$ (Fig. 2).

At the first approach, deviation of the modeled energy of recombination transition (employing the complete form of band states splitting description) from the experimental results implies that the reason of this discrepancy may be the dependence of exchange interaction constants $\left(N_{0} \alpha-N_{0} \beta / 3\right)$ on a magnetic field. To get the coincidence with the experiment, we found that exchange interaction constants should be magnetic field dependent (Fig. 3). However, this is in contradiction with the $s, p-d$ exchange interaction theory, which was formulated for constant values of exchange interaction constants [1-3]. Thus, the obtained result must be analysed with special care.

Below we analyse the possible reasons of the discrepancy between the experiment and theory, employing the complete form of band states splitting description: 


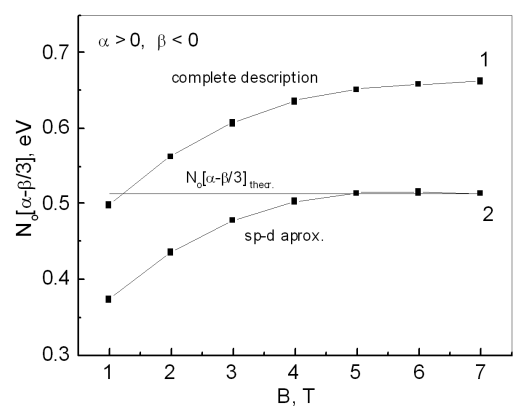

Fig. 3. Magnetic field dependence of the sum of exchange interaction constants $\left(N_{0} \alpha-N_{0} \beta / 3\right)$, assumed to get the coincidence with the experiment: 1 - in the case of complete form of band states splitting description; 2 - band states splitting approximation with the $s, p-d$ exchange interaction term only.

1. magnetic field dependence of exchange interaction constants $N_{0} \alpha-N_{0} \beta / 3$,

2 . inclusion of the Landau splitting term $\hbar \omega_{\text {ce }}$ in transition energy description.

\subsection{Exchange interaction constants at low magnetic fields}

Analysing the result of the obtained higher values of exchange constants $N_{0} \alpha-N_{0} \beta / 3$ and their dependence on magnetic field (Fig. 3), in making the decision, we relied on the fact that the $s, p-d$ exchange interaction theory was successfully applied for interpretation of magneto-optical results in SMSCs. In that case the $\hbar \omega_{\text {ce }}$ term was not accounted for in splitting of band states and constant values of $N_{0} \alpha$ and $N_{0} \beta$ were used. Inclusion of the Landau splitting term generates in the case of $\mathrm{Cd}_{0.7} \mathrm{Mn}_{0.3} \mathrm{Te}$ the increasing deviation from the experiment (Fig. 2). These experimental evidences allow to resume that determined higher values of exchange constants $N_{0} \alpha-N_{0} \beta / 3$ and their dependence on magnetic field, are not reasonable. But the dependence of exchange interaction constants $N_{0} \alpha-N_{0} \beta / 3$ on a magnetic field, obtained in the case of band states splitting approximation with $s, p-d$ exchange term, seems to be possible at low magnetic fields.

Deviations between the experiment (the Raman scattering experiments) and modeling of transition energy (by the $s, p-d$ exchange interaction term) were also observed for $\mathrm{CdMnSe}(\mathrm{S})$ [8, 9]. In the Raman study the energy of spin-flip transition $E_{\mathrm{s}-\mathrm{f}}(B)$ was measured in the same magnetic field range as studied by us. In this case the deviation between the experiment and the predictions of the $s, p-d$ exchange interaction theory was $\Delta E_{\mathrm{s}-\mathrm{f}}(B) \approx 1 \mathrm{meV}$ at magnetic field $B<1 \mathrm{~T}$ and vanished at fields of $3-4 \mathrm{~T}$. As the $s-d$ exchange interaction constant $N_{0} \alpha$ is determined from the same set of experiments at higher magnetic fields $B>3 \mathrm{~T}$, this may result in the appearance of uncertainty $\Delta E_{\mathrm{s}-\mathrm{f}}(B)$ in determination of spin-flip energy $E_{\mathrm{s}-\mathrm{f}}(B)$ at low magnetic field $B<1 \mathrm{~T}$. Authors of the mentioned experiment suggested that such discrepancy must be proportional to a differential susceptibility $\Delta E_{\mathrm{s}-\mathrm{f}}(B) \sim \partial M / \partial B$, as its value is the largest at low magnetic fields. Similar deviation $(\approx 1.2 \mathrm{meV})$ between the experiment and predictions of the $s, p-d$ exchange theory is observed by us for $\mathrm{Cd}_{0.7} \mathrm{Mn}_{0.3} \mathrm{Te}$ at magnetic field $0<B<3 \mathrm{~T}$ in the present study. We suppose that the reason of this discrepancy may be the uncertainty in $N_{0} \alpha$ determination at low magnetic fields. However, the PL measurements should allow to obtain the corrected value of exchange interaction constants $\left(N_{0} \alpha-N_{0} \beta / 3\right)$ at fields $B<5 \mathrm{~T}$ (curve 2 in Fig. 3), where the spin-flip measurements (determination of $N_{0} \alpha$ ) fail due to considerable uncertainties.

\subsection{Role of Landau splitting term $\hbar \omega_{\text {ce }}$}

We already concluded that the obtained higher values of exchange constants $N_{0} \alpha-N_{0} \beta / 3$ and their dependence on magnetic field is not justified. Further we should discuss the role of the Landau splitting term in the description of band edge states splitting in magnetic field. It is ussually assumed (and experimentally confirmed) that band edge energies of III-V and II-VI semiconductors follow the position of $n=0$ Landau levels and increases in magnetic field [10-14]. In semimagnetic semiconductors the enhanced spin splittings in magnetic field are commonly detected in reflection experiments from the position of $\sigma^{+}$and $\sigma^{-}$components of radiation in the Faraday geometry $[1,2,4,6,9]$. These results were successfully explained when the $\hbar \omega_{\text {ce }}$ term was not accounted in the band states splitting description. PL measurements of polarized components of radiation ( $\pi$ and $\sigma$ (Voigt), $\sigma^{+}$ and $\sigma^{-}$(Faraday)) allow to evaluate directly the effective band gap narrowing in magnetic field. For $\mathrm{Cd}_{0.7} \mathrm{Mn}_{0.3} \mathrm{Te}$ the red shift of effective band gap energy change in magnetic field is $3.2 \pm 0.2 \mathrm{meV} / \mathrm{T}$, as result from our $\mathrm{PL}$ experiment [15]. The same value was obtained by the authors of Ref. [16], and slightly different values of the red shift for $\mathrm{Cd}_{1-x} \mathrm{Mn}_{x}$ Te were also reported [17, 18].

Performing the analysis of $\mathrm{Cd}_{0.7} \mathrm{Mn}_{0.3} \mathrm{Te}$ luminescence results in the Voigt geometry, we determined the energies of recombination emission and had the opportunity to evaluate the values of band state splittings, involved in the process. In the case when the term $\hbar \omega_{\text {ce }}$ was included in band states splitting description, the obtained higher values of exchange constants $N_{0} \alpha-N_{0} \beta / 3$ and their dependence on magnetic field appeared to be not reasonable. This result implies that the Landau splitting term $\hbar \omega_{\text {ce }}$ should not be involved in band states splitting description in magnetic field in semimagnetic $\mathrm{Cd}_{0.7} \mathrm{Mn}_{0.3} \mathrm{Te}$, that also means that band edge energies are pinned in magnetic field and do not change their position as the $n=0$ Landau level.

After previous remarks we arrive at the main and very important question, why the Landau splitting term $\hbar \omega_{\text {ce }}$ should be included to describe dependence of band edge energies on magnetic field in III-V and II-VI semiconductors, and why the same term $\hbar \omega_{\text {ce }}$ brings in discrepancies 
in the description of transition energy in semimagnetic $\mathrm{Cd}_{0.7} \mathrm{Mn}_{0.3}$ Te. The absence of the Landau splitting term $\hbar \omega_{\text {ce }}$ in band edge splitting in magnetic field (in this case there holds the approximation by $s, p-d$ exchange interaction term) is observed in the presence of magnetic impurities in the compound. This fact allows to presume that interactions of magnetic type may influence the band edge energy value position in magnetic field.

\subsection{Influence of $M n d$ levels on the band states}

In SMSCs the exchange interactions between the $\mathrm{Mn}^{2+}$ spins of half-filled $3 d$ shell and spins of band $(s, p)$ electrons result in considerable enhancement of the Zeeman splittings in a magnetic field. $3 d^{5} \mathrm{Mn}$ states give rise to local magnetic moments with $S=5 / 2$, which hybridize strongly with $\Gamma_{8}$ valence band states, but do not mix with $\Gamma_{6}$ conduction band states, due to the symmetry reasons $[19,20]$. The hybridization strength is characterized by parameter $V_{p d}$ describing the average interaction between Mn $d$ levels and valence band states [21, 22].

To analyze the influence of $\mathrm{Mn}$ in a given II-VI material we first take into account that the $d$ levels of magnetic impurity are split into the spin-up $d^{\uparrow}$ levels (situated at $E_{\mathrm{V}}-E_{d}$ ) and spin-down levels $d^{\downarrow}$ (at the energy $\left.E_{\mathrm{V}}-E_{d}+U_{\text {eff }}\right)$, where $E_{d}$ is energy of Mn atomic $d$ level and $U_{\text {eff }}$ is polarity energy associated with adding an extra electron to $\mathrm{Mn}$ atom in a solid $[19,22]$. The occupation number of $n_{d \uparrow}$ and $n_{d \downarrow}$ is, respectively, 1 and 0 .

The influence of magnetic ions on the energy level structure of the host semiconductor was already revealed in piezomodulated reflectivity experiments in $\mathrm{Cd}_{1-x} \mathrm{Mn}_{x}$ Te and $\mathrm{Zn}_{1-x} \mathrm{Mn}_{x} \mathrm{Te}[23,24]$. Transition at $2.1-2.2 \mathrm{eV}$ in $\mathrm{Cd}_{1-x} \mathrm{Mn}_{x} \mathrm{Te}$ and at $2.3-2.4 \mathrm{eV}$ in $\mathrm{Zn}_{1-x} \mathrm{Mn}_{x}$ Te showed no observable Zeeman shift and splitting in magnetic field up to $15.6 \mathrm{~T}$ and was associated with the $\mathrm{Mn}^{2+}$ presence $[23,24]$. The $2.2 \mathrm{eV}$ transition of $\mathrm{Mn}^{2+}$ in $\mathrm{Cd}_{1-x} \mathrm{Mn}_{x}$ Te was attributed to the transition from the ${ }^{6} A_{1}\left({ }^{6} S\right)$ ground state of $3 d^{5}$ configuration to the lowest crystal-field-split ${ }^{4} T_{1}$ state, in which one of $3 d^{5}$ electrons has an antiparallel spin [25].

Following this observation we looked for the evidence of band edge energy pinning in the system studied. In coordination chemistry of $\mathrm{Mn}^{2+}$ the $3 d^{5}$ states are treated as highly localized in the ${ }^{6} A_{1}$ many-electron ground state. As already was mentioned the $\mathrm{Mn}^{2+} 3 d^{5}$ level is split into majority-spin (up-spin) and minority-spin (down-spin) levels separated by $\approx 5 \mathrm{eV}$, according to band structure calculations [26]. Under tetrahedral $\left(T_{d}\right)$ point-group symmetry each of the up-spin and down-spin $d$ levels are further split into $t_{2}$ and $e$ sublevels (Fig. 4). In the tetrahedral crystalline environment the split $d$ states of $\mathrm{Mn}$ transform as $t_{2}\left(\Gamma_{15}\right)$ and $e\left(\Gamma_{12}\right)$ representations. The anion $p$ states transform as $t_{2}\left(\Gamma_{15}\right)$ representation. Since the Mn $3 d$ e states transform differently from Te $5 p$ states, there is no $p-d$ hybridization between these states. Hybridization is possible between the Mn and Te spin-up states of the same $t_{2}$ symmetry and the interaction between them produces a lower bonding $B_{+}$and higher antibonding $A B_{+}$bands. The coupling between Mn and anion spin-down states of the same $t_{2}$ symmetry results in a lower bonding $B_{-}$and higher antibonding $A B_{-}$bands, according to Wei and Zunger [26] (Fig. 4). The confirmations of the large influence of $p-d$ hybridization on the band states are provided in experimental photoemission spectra of $\mathrm{Cd}_{1-x} \mathrm{Mn}_{x} \mathrm{Te}$ [27] and by fully hybridized band structure calculations of $\mathrm{Cd}_{0.7} \mathrm{Mn}_{0.3} \mathrm{Te}$ [28].

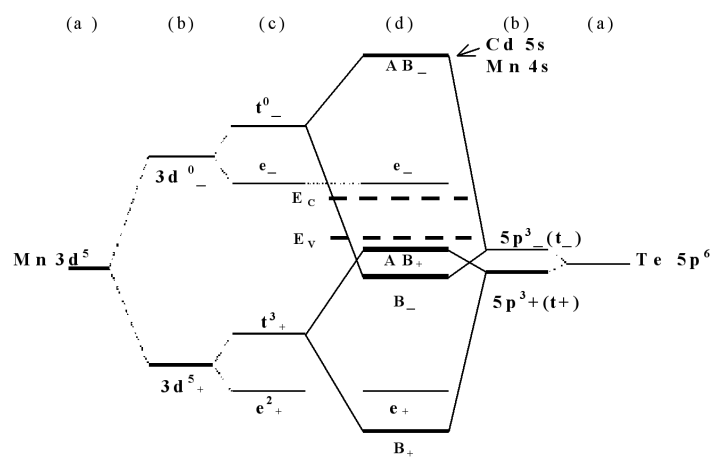

Fig. 4. $p-d$ level interaction scheme of anion and substitutional magnetic cation in ferromagnetic $\mathrm{CdMnTe}_{2}$ [26]: (a) atomic levels, (b) exchange-split atomic levels, (c) influence of the crystal field, (d) level interaction in the solid.

The interaction scheme outlined above implies that the observed pinning of band edge energies in magnetic field in $\mathrm{Cd}_{0.7} \mathrm{Mn}_{0.3}$ Te could be attributed to the influence of highly localized Mn $3 d^{5}$ levels to the band states of host semiconductor. We suppose that the pinning of band edge energies is closely related with the energy position of the hybridized Mn spin-up $3 d^{5}\left(t_{+}^{3}\right)$ and Te $5 p_{+}^{3}$ levels in the valence band and the influence of unhybridized Mn spin-down $3 d^{5}\left(e_{-}^{0}\right)$ level to $\mathrm{Cd} 5 s$ (Mn 4s) states forming the conduction band (according to hybridization scheme of Wei and Zunger for $\mathrm{CdMnTe}_{2}[26]$ ). The presence of $e_{-}$level is revealed in absorption experiments, as it emerges from the conduction band in $\mathrm{Cd}_{1-x} \mathrm{Mn}_{x} \mathrm{Te}$ for $x>0.4$ and is detected as $2.2 \mathrm{eV}$ transition responsible for absorption saturation [29]. We suppose that both mentioned levels involved in intra-center excitation, as the transition from the occupied $\mathrm{Mn} t_{+}$orbital to the lowest unoccupied $\mathrm{Mn} e_{-}$orbital [30, 26], are associated with the band energies pinning. Experimental evidences of the presence of highly localized level at $2.1 \mathrm{eV}$ in $\mathrm{Cd}_{1-x} \mathrm{Mn}_{x}$ Te, which had no Zeeman shift and splitting in magnetic field [23, 24], strongly supports the proposed explanation of band edge energies pinning in magnetic field.

This is in line with theoretical predictions. The relationship between the exchange interaction constant and hybridization parameter $V_{p d}$ determining the $p-d$ band mixing [19], follows from the Schrieffer and Wolff transformation [31]:

$$
N_{0} \beta=-2 V_{p d}^{2}\left[\left(E_{d}+U_{\text {eff }}-E_{\mathrm{V}}\right)^{-1}+\left(E_{\mathrm{V}}-E_{d}\right)^{-1}\right] .
$$


This numerical expression also implies that magnetic field dependence of exchange interaction constant is not reasonable, as the parameter $V_{p d}$ is assumed to be magnetic field independent $[21,22]$.

We resume that performed analysis of two possible reasons of the obtained discrepancy from the experiment, using the complete form of band states splitting description - the magnetic field dependence of exchange interaction constants or band edge energies pinning in magnetic field - proved validity of the latter.

We have already concluded that the Landau splitting term $\hbar \omega_{\text {ce }}$ can be omitted in the description of band edge states splitting in magnetic field in $\mathrm{Cd}_{0.7} \mathrm{Mn}_{0.3} \mathrm{Te}$ and possibly in other Mn-based large gap SMSCs. This experimental result also means that the band edge splitting scheme in magnetic field, illustrating the influence of the $s, p-d$ exchange, as proposed in Ref. [6], p. R45, is not confirmed experimentally.

\section{Conclusions}

Even formally the energy of $\mathrm{Cd}_{0.7} \mathrm{Mn}_{0.3} \mathrm{Te}$ luminescent transition could be described using the model of magnetic field dependent exchange interaction constants, we conclude that discrepancy between the model and the experiment results from the influence of Mn-related $d$ states on the host band states. Results of the performed magneto-PL analysis indicate that the Landau splitting term $\hbar \omega_{\text {ce }}$ can be omitted in the description of band edge splitting in magnetic field in $\mathrm{Cd}_{0.7} \mathrm{Mn}_{0.3} \mathrm{Te}$. The absence of the Landau splitting term $\hbar \omega_{\text {ce }}$ means that band edge energies are pinned in a magnetic field. The pinning is closely related with the presence of highly localized $3 d^{5}$ levels of $\mathrm{Mn}^{2+}$ and the hybridization of these levels with the band states of host semiconductor. The proposed explanation of the band edge energies pinning in magnetic field also supports the description of band states splitting with the $s, p-d$ exchange term only, taking into account that based on this approximation the $s, p-d$ exchange interaction theory was applied to SMSCs.

Results of magneto-PL measurements in magnetic field allow to obtain the corrected values of exchange interaction constants $\left(N_{0} \alpha-N_{0} \beta / 3\right)$ at magnetic fields $B<5 \mathrm{~T}$ in SMSCs.

\section{References}

[1] A. Gaj, J. Ginter, R.R. Galazka, Phys. Status Solidi B 89, 655 (1978).

[2] J.A. Gaj, R. Planel, G. Fishman, Solid State Commun. 29, 435 (1979).

[3] J.A. Gaj, P. Byszewski, M. Cieplak, G. Fishman, R.R. Galazka, J. Ginter, M. Nawrocki, N.T. Khoi, R. Planel, R. Ranvaud, in: Inst. Phys. Conf. Ser. 43, 1113 (1979).

[4] N.B. Brandt, V.V. Moshchalkov, Adv. Phys. 33, 193 (1984).
[5] R. Brazis, L. Barauskaitè, V. Ivanov, M. Godlewski, Acta Phys. Pol. A 109, 731 (2006).

[6] J.K. Furdyna, J. Appl. Phys. 64, R29 (1988).

[7] J.O. Goede, W. Heimbrodt, Phys. Status Solidi B 146, 11 (1988).

[8] D. Heiman, P.A. Wolff, J. Warnock, Phys. Rev. B 27, 4848 (1983).

[9] D. Heiman, Y. Shapira, S. Foner, Solid State Commun. 45, 899 (1983).

[10] E. Burstein, G.S. Picus, H.A. Gebbie, F. Blatt, Phys. Rev. 103, 826 (1956).

[11] S. Zwerdling , R.I. Keyes, S. Foner, H.H. Kolm, B. Lax, Phys. Rev. 104, 1805 (1956).

[12] Landau Level Spectroscopy. Modern Problems in Condensed Matter Sciences, Vol. 27.1., Eds. G. Landwehr, E.I. Rashba, Elsevier, Amsterdam 1991, p. 398.

[13] G. Landwehr, High Magnetic Fields in Semiconductor Physics. II. Transport and Optics, Springer, Berlin 1989, p. 283.

[14] W. Ossau, E. Bangert, G. Landwehr, J. Lumin. 40-41, 729 (1988).

[15] L. Barauskaitè, R. Brazis, V. Ivanov, M. Godlewski, Lith. J. Phys. 49, 267 (2009).

[16] E.D. Isaacs, D. Heiman, J.J. Zayhowski, R.N. Bicknell, J.F. Schetzina, Appl. Phys. Lett. 48, 275 (1986).

[17] W. Hayes, K.S. Wong, J.F. Ryan, A.K. Ramdas, J. Lumin. 40-41, 72 (1988).

[18] W. Heiss, G. Prechtl, G. Springholz, Phys. Rev. B 63, 165323 (2001).

[19] A.K. Bhattacharjee, G. Fishman, B. Coqblin, Physica 117\&118, 448 (1983).

[20] K.C. Haas, B.E. Larson, H. Ehrenreich, A.E. Carlsson, J. Magn. Magn. Mater. 54-57, 1283 (1986).

[21] B.E. Larson, K.C. Haas, H. Ehrenreich, A.E. Carlsson, Solid. State Commun. 56, 347 (1985).

[22] B.E. Larson, K.C. Haas, H. Ehrenreich, A.E. Carlsson, Phys. Rev. B 37, 4137 (1988).

[23] Y.R. Lee, A.K. Ramdas, R.L. Aggarwall, Phys. Rev. B 33, 7383 (1986).

[24] Y.R. Lee, A.K. Ramdas, R.L. Aggarwall, Phys. Rev. B 38, 10600 (1988).

[25] R.Y. Tao, M.M. Moriwaki, W.M. Becker, R.R. Galazka, J. Appl. Phys. 59, 3772 (1983).

[26] Su-Huai Wei, A. Zunger, Phys. Rev. B 35, 2340 (1987).

[27] L. Ley, M. Taniguchi, J. Ghijsen, R.L. Johnson, A. Fujimori, Phys. Rev. B 35, 2839 (1987).

[28] K.C. Hass, H. Ehrenreich, J. Cryst. Growth 86, 8 (1988).

[29] N.T. Khoi, J.A. Gaj, Phys. Status Solidi B 83, K133 (1977).

[30] V.F. Aguekian, N.N. Vasil'ev, A.Yu. Serov, N.G. Filosofov, J. Cryst. Growth 214-215, 391 (2000).

[31] J.R. Schrieffer, P.A. Wolff, Phys. Rev. 149, 491 (1966). 